\title{
Bronchoscopy and the peripheral nodule in the age of lung cancer screening and targeted therapies
}

\author{
William Krimsky • Luis M. Seijo
}

Published online: 25 January 2012

(C) Springer Science+Business Media, LLC 2012

\begin{abstract}
With the publication of the national lung cancer screening trial (NLST), screening for lung cancer is expected to become more commonplace. The discovery of pulmonary nodules that require further management will, therefore, increase as well and will generate a growing need for tissue given the recent inroads in the pursuit of individualized therapies for lung cancer. This, coupled with imaging advances in screening process, have all conspired to raise the bar on the limits of the bronchoscope, especially in the pursuit of small peripheral lesions. This review highlights advances in navigational bronchoscopy and tissue processing which may help the bronchoscopist of the 21 st century meet these and other challenges.
\end{abstract}

Keywords Navigational bronchoscopy · Lung cancer screening · Pulmonary nodules · Transbronchial biopsy . Interventional pulmonology

\section{Introduction}

Since the introduction of the flexible bronchoscope by Shigeto Ikeda in the 1960s and more recently endobronchial ultrasound, few technological advances have improved the diagnostic yield of conventional bronchoscopy. The peripheral

\section{W. Krimsky}

Harry and Jeanette Weinberg Cancer Center,

Franklin Square Hospital,

Baltimore, MD, USA

\section{M. Seijo $(\bowtie)$}

Servicio de Neumología, IIS-Fundación Jimenez Díaz-CIBERES,

Avenida de los Reyes Católicos 2,

Madrid 28040, Spain

e-mail: luis.seijo@fjd.es nodule is particularly challenging for the bronchoscopist, whereas improvements in the endoscopic sampling of the mediastinum have successfully consolidated the role of the bronchoscope in lung cancer staging. An ever-growing need for tissue as well as significant inroads in the pursuit of individualized therapies for lung cancer coupled with imaging advances in lung cancer screening have all conspired to raise the bar on the limits of the bronchoscope, especially in the pursuit of small peripheral lesions. This review highlights advances in navigational bronchoscopy and tissue processing which may help the bronchoscopist of the 21 st century meet these and other challenges.

\section{Navigational bronchoscopy}

Endoscopic exploration of the tracheobronchial tree began in 1897, with the first endoscopic removal of a foreign body by Gustav Killian [1]. As with other modalities, bronchoscopy and its associated techniques have evolved over time from a mere tube with a mirror and lens to the advanced flexible fiber optic scope in use today [1,2]. Modern bronchoscopic techniques, such as endobronchial ultrasound [3-5], along with improvements in the imaging quality of the bronchoscope itself have expanded the scope of the procedure as well as improved diagnostic yield in a variety of circumstances.

Navigational technologies in the tracheobronchial tree were developed to further expand the reach of flexible bronchoscopy. Originally designed to improve diagnostic yield rates of bronchoscopy with respect to peripheral lesions, these technologies typically, but not always, work in tandem with the conventional bronchoscope. Given the limited access of the standard bronchoscope to the majority of the tracheobronchial tree, navigational bronchoscopy attempts to improve upon this limitation by using a bronchoscope in conjunction 
with a predetermined pathway through the tracheobronchial tree employing the airways as the conduit to the lesion. This can be accomplished in a variety of ways and there are now several navigational technologies on the market.

The yield of conventional bronchoscopy in the diagnosis of parenchymal or peripheral lesions, regardless of size, is roughly $60 \%$ [6]. However, for lesions $<2 \mathrm{~cm}$ in size, the yield drops substantially to approximately $23 \%$ [6]. Lesions of this size that are more peripherally located are even harder to diagnose with yields approximating 14\%-35\% $[6,7]$. Therefore, attempting to locate a small lesion beyond the visual range of the conventional bronchoscope without guidance is often a time consuming and difficult task. In essence, the combination of size, operator skill, and a limited visual access to the tracheobronchial tree, all conspire to limit the yield rates of standard bronchoscopy in this setting [1, 6-8]. Navigational bronchoscopy was designed to address these shortcomings through both hardware and softwarebased solutions [9].

At present there are, or soon will be, four commercially available navigational systems on the market. These include the Lung Point system (Bronchus Technologies, Inc.), the Bf Navi system (Olympus, Inc.), the Spin Drive system (Veran, Inc.), and the i-Logic system (superDimension, Inc.). They all involve mapping of the airways followed by a designated or planned pathway to the lesion. The specifics of the individual methods of localization and navigation are beyond the scope of this review. Briefly, Bronchus and Olympus focus on a visual or virtual approach to navigation without real-time feedback, while Veran and superDimension rely on additional hardware by utilizing a steerable sensor or sensors that provide feedback to the user with respect to instrument location within the tracheobronchial tree. Published data regarding diagnostic yield of navigational bronchoscopy are available for the superDimension system only because it has been available for several years. Preliminary yields with the other systems appear to match [10] those observed with the superDimension system, although such data are limited.

In general, the diagnostic yield of navigational bronchoscopy is significantly higher when compared with traditional bronchoscopy in the diagnosis of peripheral lesions. One of the first studies to evaluate the performance of the superDimension navigation technology reported a diagnostic yield of $70 \%$ for lesions less than $4 \mathrm{~cm}[11 \bullet \cdot]$. Navigation bronchoscopy was diagnostic in $100 \%$ of lesions greater than $4 \mathrm{~cm}$ in size, although the number of patients studied was small. Interestingly, yields did not diminish for lesions $<2 \mathrm{~cm}$ remaining at $70 \%$ (14/ 20) while diagnostic yield for lesions between 2 and $4 \mathrm{~cm}$ was $71.4 \%(15 / 21)$. In essence, lesion size did not contribute significantly to differences in yield rates [10]. This is in contrast to the trend associated with traditional bronchoscopy.

Several studies have achieved roughly equivalent results. One such study that looked at 40 peripheral lesions evaluated other variables that might contribute to variations in diagnostic yield, including distance from the pleural surface, distance to the target, location, and operator experience among others. Average lesion size in the study was $23 \mathrm{~mm}$. Overall, the diagnostic yield was $63 \%$ [12]. Modest improvements in yield with larger lesions were seen but not as impressive as those typical of conventional bronchoscopy. The authors also noted that yields varied significantly with the calculated CT to body divergence which is essentially a measure of variation or uncertainty regarding the position of the steerable probe. Diagnostic yield when the CT to body divergence was $<4 \mathrm{~mm}$ was $77 \%$ compared with $44 \%$ in interventions with a $\mathrm{CT}$ to body divergence $>4 \mathrm{~mm}$. A subsequent study of 89 lesions, with an average size of $24 \mathrm{~mm}$, also evaluated the time required for these procedures [13]. The authors found that the procedure lasted anywhere from 16 to $45 \mathrm{~min}$ with diagnostic yields of $67 \%$, making navigational bronchoscopy sometimes more time consuming than conventional bronchoscopy. It should be noted that complication rates reported in these studies were no greater than historical controls and in some cases better.

A multimodality approach combining navigational bronchoscopy with radial endobronchial ultrasound (EBUS) has been studied in a prospective randomized controlled study of 120 patients [14•]. Patients were allocated to one of three study arms: endobronchial ultrasound only, electromagnetic navigational bronchoscopy only (ENB), or the combined procedure. Yield rates were $69 \%(27 / 39)$ in the EBUS group, $59 \%(23 / 39)$ in the ENB group, and $88 \%(35 / 40)$ in the combined EBUS+ENB group. The diagnostic yield of the combined approach was also independent of lesion size or lobar distribution. Improved precision with the combined procedure was attributed to ultrasound-guided repositioning of the catheter following successful navigation to the vicinity of the lesion.

Considerable uncertainty persists regarding different variables associated with the procedure such as the type and variety of tissue sampling tools employed, the value of fluoroscopy, and the presence of a bronchus sign [15]. Biopsy forceps have been employed in the vast majority of these studies and in some cases were the only tool used to obtain tissue. Therefore, it is difficult to ascertain whether additional tools or a specific sampling sequence might modify overall yields. The use of fluoroscopy is also an area of uncertainty. Fluoroscopy is usually employed not as a navigational tool, but rather to assess tool displacement. Some studies, though, found no difference in yield with or without fluoroscopy $[12,15]$. Finally, emerging work with respect to lesional characteristics seems to have further refined reliability in these settings with respect to yield. The presence of a bronchus sign, which is a visible airway leading to the lesion on the virtual renderings of the tracheobronchial tree, suggests a significant improvement in diagnostic yield compared to those lesions without this finding. A recent prospective study of 51 patients demonstrated yield rates 
of $79 \%(30 / 38)$ in lesions with a bronchus sign versus a yield of $31 \%$ for lesions without an identifiable bronchus sign (4/13).

Bronchoscopy and targeted therapies

Bronchoscopists currently face the challenge of providing not only a diagnosis, but also adequate tissue samples for ancillary studies. Such studies are becoming increasingly complex since they rely on molecular analysis of mutations, immunohistochemistry, and in situ hybridization, to name a few, and have been designed to facilitate individualized treatment, especially for patients with lung cancer. A growing body of evidence suggests that cytological samples or even stained slides, when processed adequately, can yield sufficient material for adequate mutation analysis without the need for a more invasive approach, thus facilitating the appropriate choice of chemotherapy in some patients. Cytological samples are often the only material which a bronchoscopist can provide, because forceps biopsy or needle aspiration yield small samples often consisting of a limited number of malignant cells.

Although initial reports suggested that cytological samples were inadequate [16], recent findings have shown that minimally invasive techniques might be sufficient [17-19], [20•]. Rapid on-site evaluation (ROSE) in the bronchoscopy suite may be instrumental in such cases in order to guarantee that the sample obtained is adequate for both a diagnosis and mutation analysis in the majority of cases. A skilled cytopathologist can review existing Papanicolaou-stained slides in order to select the best samples for molecular analysis. In general, a slide is considered adequate when at least $50 \%$ of the cellularity is composed of malignant cells. Selected Papanicolaou-stained smears can be rinsed in alcohol and need not be destained prior to DNA extraction.

Routine DNA extraction from stained slides and/or cytological samples obtained during bronchoscopy is feasible and yields results comparable to mutation analysis of paraffinembedded or frozen tissue [21]. Such results match those previously reported from biopsy series with respect to the frequency and distribution of EGFR mutations by gender, smoking history, histology, and TKI activity [22-24, 25••]. Smouse et al. [26] have shown that cell blocks may yield even better sensitivity when compared with surgical specimens, suggesting that the adequacy of a sample depends on its quality and cellularity rather than the technique used to obtain it. A few small series have assessed mutations in exons 19 and 21 of the EGFR gene in cytological samples, including stained smears [27-29]. Fukui et al. [30] reported better results using PCR analysis of stained cytology slides rather than biopsy specimens in a series of 92 patients. The difference was attributed to DNA preservation rather than the sample's origin or cellularity. Finally, Oshita et al. [31] assessed clinical activity of gefitinib in 26 patients, confirming a $91 \%$ response rate in 11 patients using a novel heteroduplex method to study mutations in exons 19 and 21 of the EGFR gene. The samples were obtained by transbronchial abrasion [31].

Multi-gene mutation analysis can also be performed in EBUS-TBNA samples obtained from mediastinal lymph nodes [32]. In one study using frozen tissue cell blocks obtained with EBUS-TBNA, EGFR gene mutations were detected in $26.9 \%$ of patients, with an overall response rate to gefitinib of $54.5 \%$ and disease control rate of $86.4 \%$. K-ras gene mutations were rare $(3.5 \%)$, but $p 53$ gene mutations were common, detected in 47 cases $(41.6 \%)$. As expected, patients with $p 53$ mutations were refractory to chemotherapy and had a poor prognosis despite treatment. The authors had previously reported their experience with paraffin-embedded tissue, but concluded that frozen samples yield better results and less false positives because of improved cellularity and better preservation of DNA [33].

The bronchoscopist should know that assessment of EGFR mutations in exons 18, 19, 20, and 21, ALK, p53, and K-ras mutations in cytological samples or even stained slides is feasible in NSCLC patients. Identification of EGFR mutations in bronchoscopy samples predicts clinical response to targeted therapies, thus extending the benefits of individualized treatment selection to NSCLC patients from which tumor biopsies are not available and expanding the role of the bronchoscopist in the diagnosis and management of patients with NSCLC.

\section{Lung cancer screening}

Current advances in lung cancer screening also pose a challenge to bronchoscopists frequently accustomed to diagnosing patients with advanced-stage lung cancer, large tumors, and/or mediastinal lymphadenopathy amenable to conventional bronchoscopic techniques. Until recently, lung cancer screening had been discouraged based on evidence accrued more than 30 years ago from several randomized trials [34, 35]. Recent technological advances, however, have renewed interest in lung cancer screening. Pioneers of CT-based screening showed in 1999 that such screening is feasible and effective [36]. Their efforts have led to the design of several trials aimed at determining whether early lung cancer detection is possible using low-dose CT (LDCT) and whether it can improve survival in this dreaded disease. Such efforts have culminated in the National Lung Cancer Screening Trial (NLST), which demonstrated a survival benefit with LDCT screening at the expense of a debatable rise in false-positive screening results $[37 \bullet \bullet]$.

\section{Low-dose CT}

Several studies have confirmed LDCT's ability to detect small pulmonary nodules and therefore lung cancer at an early stage. ELCAP screened 1000 men annually with LDCT. All nodules found on baseline or subsequent LDCTs were worked up with 
strict adherence to a pre-determined protocol designed to detect growth. More than $20 \%$ of individuals had noncalcified lung nodules on baseline screening which required one or more follow-up studies at specified intervals. Growth was considered key in order to avoid unnecessary biopsies which were eventually obtained for 28 non-calcified nodules, 27 of which were proven to be lung cancer. Only one biopsy resulted in a benign diagnosis, and the majority of cancers were stage IA (81\%) [36]. Eight additional nodules were biopsied during subsequent rounds of screening because they were shown to grow on a follow-up LDCT. Seven were lung cancer and one was a benign lesion. Of the six non-small cell lung cancers, five were stage IA and only one was advanced stage [38]. Data from these preliminary studies demonstrated that screening for lung cancer using LDCT is effective in diagnosing early-stage lung cancer and results in few invasive diagnostic procedures for benign disease.

Subsequent studies from the same group incorporating more than 60 centers worldwide have confirmed preliminary results [39]. Recent experience with LDCT has led to refined protocols. In brief, initial baseline screening is considered positive if it detects non-calcified nodules larger than $5 \mathrm{~mm}$. Annual screening is considered positive if any new nodule is detected. Work up depends on the size of the nodule, the majority of which are subject to follow-up with repeat LDCT at specific time intervals. A biopsy is generally recommended only when a large non-calcified nodule $(>15 \mathrm{~mm})$ is found on the baseline LDCT or an existing nodule is growing. The i-ELCAP investigators reported diagnosing more than 400 lung cancers after almost 60,000 rounds of screening in more than 30,000 individuals, $85 \%$ of which were stage I. The 10 -year estimated survival rate of individuals with lung cancer was $80 \%$. Patients with stage IA lung cancer undergoing surgical resection within a month of the diagnosis had a 10-year expected survival of $92 \%$.

Experience with LDCT screening has raised several concerns, chief among them, the lack of a control group, overdiagnosis, and false-positive outcomes associated with an increased risk of costly and potentially dangerous work up. The NLST has addressed the first concern proving that mortality in a control group is greater than that seen in a screened population, while abundant epidemiological, clinical, and pathological evidence demonstrates that significant overdiagnosis of lung cancer is unlikely [40]. Evidence from autopsy series, for example, reveals that incidental lung cancer is rare and may be found in less than $3 \%$ of patients at autopsy. Furthermore, most cancers found at autopsy $(>50 \%)$ are associated with regional and/or distant metastases and therefore cannot be considered clinically insignificant. Finally, the vast majority of untreated patients with screening-detected early-stage lung cancer have died within 5 years of the diagnosis (personal communication, i-ELCAP investigators).
A greater concern for bronchoscopists is the likelihood of false-positive results from screening. LDCT may be associated with cumulative false-positive rates in excess of 30\% [41]. While findings from two large screening trials including i-ELCAP and the NELSON trial in Europe have shown lower false-positive rates than the NLST, bronchoscopists can expect to be called upon to diagnose small pulmonary nodules or at least attempt to rule out malignancy in a growing number of patients if lung cancer screening becomes a reality [36, 42]. Conservative estimates suggest that less than $0.1 \%$ of screening rounds will result in an invasive procedure for benign disease [36]. However, nodule detection and follow-up in the context of a well-designed trial is far from what can be expected in the real world, where chest $\mathrm{CT}$ has become almost as commonplace as conventional chest radiography. Judging from the NLST results, bronchoscopists can expect to be called upon to diagnose such nodules. The current size limit of what is considered suspicious approximates $1 \mathrm{~cm}$. In the NELSON trial, a test is considered positive if a nodule of at least $9.8 \mathrm{~mm}$ is found, or if a smaller nodule shows significant growth by volumetric analysis on follow-up LDCT [42]. Increasingly, the pursuit of smaller pulmonary nodules will test the limits of the bronchoscope and represent a challenge which bronchoscopists must continue to meet.

\section{Conclusions}

Given the accumulating data on lung cancer screening, the bronchoscopist will be called upon to provide real and reproducible results not only from a diagnostic standpoint but also as treatment evolves toward a more targeted approach to cancer therapy.

Disclosure W. Krimsky: consultant to CSA, Superdimension, and Olympus; L. M. Seijo: honoraria from Superdimension.

\section{References}

Recently published papers of interest have been highlighted as

- Of importance

•- Of major importance

1. Heinrich B.D.: "A Short History of Bronchoscopy." Introduction to Bronchoscopy. Edited by Armin Ernst and Harvard Medical School/ Beth Israel Deaconness Medical Center. Cambridge University Press 2004, 3.

2. Zavala DC. Diagnostic fiberoptic bronchoscopy: Techniques and results of biopsy in 600 patients. Chest. 1975;68:12-9.

3. Hearth FJF, Ernst A, Eberhardt P, et al. Endobronchial ultrasoundguided transbronchial need aspiration of lymph nodes in the radiologically normal mediastinum. Eur Respir J. 2006;28:910-4. 
4. Yasufuku K, Chiyo M, Sekine Y, et al. Real-time endobronchial ultrasound-guided transbronchaial needle aspiration of mediastinal and hilar lymph nodes. Chest. 2004;126:122-8.

5. Rintoul RC, Skwarski KM, Murchison JT, et al. Endobronchial and endoscopic ultrasound-guided real-time fine-needle aspiration for mediastinal staging. Eur Respir J. 2004;25:416-21.

6. Baaklini WA, Reinoso MA, Gorin AB, et al. Diagnostic yield of fiberoptic bronchoscopy in evaluating solitary pulmonary nodules. Chest. 2000;117:1049-54.

7. Schwarz Y, Greif J, Becker H, et al. Real-time electromagnetic navigation bronchoscopy to peripheral lung lesions using overlait CT images: the first human study. Chest. 2006;129:988-94.

8. Davoudi M, Osann K, Colt HG. Validation of two instruments to assess technical bronchoscopic skill using virtual reality simulation. Respiration. 2007;96:92-101.

9. Edell E, Krier-Morrow D. Navigational bronchoscopy: overview of technology and pratical considerations - new current procedural terminology codes effective 2010. Chest. 2010;137:450-4.

10. Eberhardt R, Kahm N, Gompelmann D, et al. LungPoint-A new approach to peripheral lesions. J Thorac Oncol. 2010;5:1559-63.

11. •• Gildea TR, Mazzone PJ, Karnak D: Electromagnetic navigation bronchoscopy a prospective study. Am J Respir Crit Care Med $2006 ; 174: 982-989$. A widely cited paper on electromagnetic navigational bronchoscopy demonstrating that navigational bronchoscopy can be an effective means of diagnosing peripheral lung lesions.

12. Markis D, Scherpereel A, Leroy S, et al. Electromagnetic navigation diagnostic bronchoscopy for small peripheral lung lesions. Eur Respir J. 2007;29:1187-92.

13. Eberhardt R, Anantham D, Herth F, et al. Electromagnetic navigation diagnostic bronchoscopy in peripheral lung lesions. Chest. 2007;131(6):1800-5.

14. - Eberhardt R, Anantham D, Ernst A, et al. Multimodality bronchoscoptic diagnosis of peripheral lung lesions. Am J Respir Crit Care Med 2007; 176:36-41. This article highlights the value of combining EBUS and ENB relative to increasing the bronchoscopic yield rates in the diagnosis of peripheral lung lesions.

15. Seijo LM, de Torres JP, Lozano MD, et al. Diagnostic yield of electromagnetic navigation bronchoscopy is highly dependent on the presence of a Bronchus sign on CT imaging: results from a prospective study. Chest. 2010;138(6):1316-21.

16. Sequist LV, Bell DW, Lynch TJ, Haber DA. Molecular predictors of response to epidermal growth factor receptor antagonists in nonsmall-cell lung cancer. J Clin Oncol. 2007;25:587-95.

17. Wong M, Yasufuku K, Nakajima T, et al. Endobronchial ultrasound: new insight for the diagnosis of sarcoidosis. Eur Respir J. 2007;29:1182-6.

18. Hearth FJ, Krasnik M, Vilmann P. EBUS-TBNA for the diagnosis and staging of lung cancer. Endoscopy. 2006;38(S1):S101-5.

19. Dooms C, Seijo L, Gasparini R, et al. Diagnostic bronchoscopy: state of the art. Eur Respir. 2010;19(17):229-36.

20. - Sequist LV, Engelman JA, Lynch TJ. Toward noninvasive genomic screening of lung cancer patients. J Clin Oncol 2009; 27:2589-2591. Molecular testing, especially of the EGFR mutation with its associated treatment, can be an affordable and accurate metric in the analysis of tissue specimens.

21. Lozano MD, Zulueta JJ, Echeveste JI, et al. Assessment of epidermal growth factor receptor and K-ras mutation status in cytological stained smears of non-small-cell lung cancer patients: correlation with clinical outcomes. Oncologist. 2011;16:877-85.

22. Lynch TJ, Bell DW, Sordella R, et al. Activating mutations in the epidermal growth factor receptor underlying responsiveness of nonsmall-cell lung cancer to gefitinib. N Engl J Med. 2004;350:2129-39.

23. Paez JG, Janne PA, Lee JC, et al. EGFR mutations in lung cancer: correlation with clinical response to gefitinib therapy. Science. 2004:304:1497-500.
24. Pao W, Miller V, Zakowski M, et al. EGFR receptor gene mutations are common in lung cancers from "never smopkers" and are associated with sensitivity of tumors to gefitinib and erlotinib. Proc Natl Acad Sci USA. 2004;101:13306-11.

25. •• Rosell R, Moran T, Queralt C, et al.: Screening for epidermal growth factor receptor mutations in lung cancer. N Engl J Med 2009; 361:958-967. This paper highlights the value of large-scale screening for EGFR mutations in patients with lung cancer in the context of the impact on care.

26. Smouse JH, Cibas ES, Janne PA, et al. EGFR mutations are detected comparably in cytologic and surgical pathology specimens of nonsmall-cell lung cancer. Cancer Cytopathol. 2009;117:67-72.

27. Boldrini L, Gisfredi S, Ursino S, et al. Mutational analysis in cytological specimens of advanced lung adenocarcinoma: a sensitive method for molecular diagnosis. J Thorac Oncol. 2007;2:1086-90.

28. Nomoto K, Tsuta K, Takano T, et al. Detection of EGFR mutations in archived cytologic specimens of non-small cell lung cancer using high-resolution melting analysis. Am J Clin Pathol. 2006;126:608-15.

29. Smith GD, Chadwick BE, Willmore-Payne C, Bentz JS. Detection of epidermal growth factor receptor gene mutations in cytology specimens from patients with non-small cell lung cancer utilizing highresolution melting amplicon analysis. J Clin Pathol. 2008;61:487-93.

30. Fukui T, Ohe Y, Tsuta K, et al. Prospective study of the accuracy of EGFR mutational analysis by high-resolution melting analysis in small samples obtained from patients with non-small cell lung cancer. Clin Cancer Res. 2008;14:4751-7.

31. Oshita F, Matsukuma S, Yoshihara M, et al. Novel heteroduplex method using small cytology specimens with a remarkably high success rate for analysing EGFR gene mutations with a significant correlation to gefitinib efficacy in non-small-cell lung cancer. $\mathrm{Br} \mathrm{J}$ Cancer. 2006;95:1070-5.

32. Takahiro N, Kazuhiro Y, Akira N, et al. EGFR, K-ras, p53 gene mutation analysis in non-small-cell lung cancer using microcaples obtained by endobroncial ultrasound-guided transbronchial needle aspiration. Chest. 2010;138:178A.

33. Takahiro N, Kazuhiro Y, Makoto S, et al. Assessment of epidermal growth factor receptor mutation by endobronchial ultrasound-guided transbronchial needle aspiration. Chest. 2007;132:591-602.

34. Fontana RS, Sanderson DR, Woolner LB, et al. Lung cancer screening: the Mayo program. Journal of Occupational Medicine. 1986;28:746-50.

35. Tockman M. Survival and mortality from lung cancer in a screened population. The John Hopkins study. Chest. 1986;89:325S.

36. Henschke CI, McCauley DI, Yankelevitz DF, et al. Early Lung Cancer Action Project: overall design and findings from baseline screening. Lancet. 1999;354:99-105.

37. •• The National Lung Screening Trial Research Team: Reduced lung-cancer mortality with low-dose computed tomographic screening. N Engl J Med 2011; 365:395-409. This recent publication has set the standard around low-dose CT scanning, in the appropriate population, to detect early-stage lung cancers with an attendant impact on mortality.

38. Henschke CI, Naidich DP, Yankelevitz DF, et al. Early lung cancer action project: initial findings on repeat screenings. Cancer. 2001;92:153-9.

39. Henschke CI, Yankelevitz DF, Libby DM, et al. Source survival of patients with stage I lung cancer detected on CT screening. N Engl J Med. 2001;355:1763-71.

40. Strauss G. Randomized population trials and screening for lung cancer breaking the cure barrier. Cancer. 2000;89:2399-421.

41. Croswell JM, Baker SG, Marcus PM, et al. Cumulative Incidence of False-Positive Test Results in Lung Cancer Screening. Ann Intern Med. 2010;152:505-12.

42. van Klaveren RJ, Oudkerk M, Prokop M, et al. Management of lung nodules detected by volume CT scanning. N Engl J Med. 2009;361:2221-9. 